\title{
Erratum
}

\section{Highly Diastereoselective Construction of Substituted Pyrrolidines: Formal Synthesis of (-)-Bulgecinine}

Biswanath Das, * Duddukuri Nandan Kumar Synlett 2011, 1285.

In the advance online publication (e-First) version of this article a wrong reference was cited in the caption of Scheme 2. The last item should be (n) ref. 5e instead of (n) ref. 4e. This has been corrected for both the current online version and the print publication. The authors apologize for this error. 